\title{
SAC- OCDMA System Using Different Detection Techniques
}

\author{
Somaya A. Abd El Mottaleb ${ }^{1}$, Heba A. Fayed ${ }^{1}$, Ahmed Abd El Aziz ${ }^{1}$ and \\ Moustafa H.Aly ${ }^{2}$ \\ I'Electronics and Communication, College of Engineering and Technology/ Arab Academy for Science, \\ Technology and Maritime Transport, Alexandria, Egypt) \\ ${ }^{2}$ (OSA Member $)$
}

\begin{abstract}
In this paper, we investigate the optimum received power for spectral amplitude coding optical code division multiple access (SAC-OCDMA) at different transmission distances using modified-AND subtraction detection technique and single photodiode (SPD) detection technique. We also investigate the effect of avalanche photodiode (APD) gain on the received power. Simulation results show that: 1) the received power decreases while the bit error rate (BER) increases with the transmission distance, and 2) both the received power and the BER increase with the APD gain for the same transmission distance.
\end{abstract}

Keywords: APD, modified-AND subtraction detection technique, phase induced intensity noise (PIIN), SAC$O C D M A, S P D$

\section{INTRODUCTION}

Recently, optical code division multiple access (OCDMA) technique is getting more attractive due to its several features as its ability to support asynchronous access, bursty traffic, secure transmission and effective cost $[1,2]$. The main drawback of OCDMA is that its performance and capacity are limited by multiple access interference (MAI) [3, 4]. Researchers proposed spectral amplitude coding (SAC) from all types of OCDMA due to its ability in restrain MAI when using a suitable detection technique at the receiver with fixed in-phase cross correlation codes [5]. Low cost broadband sources such as light emitting diodes (LEDs) can be used for SAC-OCDMA system. However, the performance of SAC-OCDMA is still limited by phase induced intensity noise (PIIN) that results from using broadband light sources [6]. Modified-AND subtraction detection technique and SPD detection technique are used for reducing PIIN and MAI [7].

In this work, enhanced double weight codes (EDW) are used as the signature codes for SAC-OCDMA systems with modified-AND subtraction detection and SPD detection techniques when APDs are used.

The remainder of this paper is organized as follows. In Sec 2, we give a detailed explanation about the detection techniques, followed by the network simulation setup in Sec.3. Section 4 is devoted for the results and discussion. Finally, the conclusion of the paper is provided in Sec.5.

\section{1}

\section{DETECTION TECHNIQUES}

\section{MODIFIED-AND SUBTRACTION DETECTION}

The SAC-OCDMA receiver diagram of this technique is shown in Fig. 1 [7]. The received optical signal is split by splitter 1 into two parts, one to the upper decoding branches and the other to the AND decoder through an attenuator. The attenuator ensures that, the interference signal has an equivalent power incident on each photo detector in the case of an inactive user. The decoder filters are placed in a parallel configuration. This structure divides the spectrum of the decoded signals. It is worth to note that, both splitters (1 and 2) and the attenuator could be replaced by a single coupler with an appropriate coupling ratio in order to get a more cost-effective receiver. The decoder has a spectral response matched to the active user, whereas the AND decoder has overlapped bins from different interferers. These overlapped bins can be represented mathematically by AND operation between the active user and interferers [8]. This technique can be performed using the inexpensive fiber Bragg-gratings (FBGs) to decode the received signal because of their low insertion losses, good spectral resolution, small size, and light weight [10]. The photo detector is composed of two photodiodes (PD and s-PD) which are connected electrically in opposition. The output signal is proportional to the power difference of the two optical inputs. In the presence of an interferer, the difference between the two signals is cancelled out. The output signal is then low-pass filtered to reduce the out-of-band high-frequency noise. After the decision circuit, the original data is restored. 


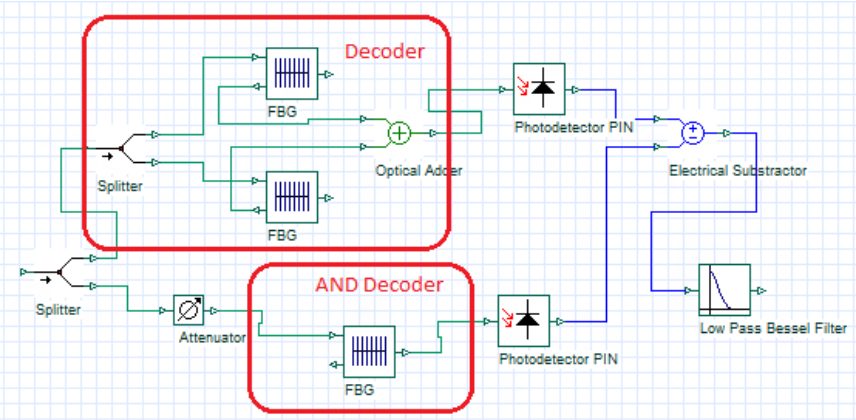

Figure 1 Modified-AND subtraction detection.

\subsection{SINGLE PHOTODIODE DETECTION}

The structure of SAC-OCDMA receiver diagram using EDW code is illustrated in Fig. 2 [10]. The incoming signal is decoded using the same spectral response of the encoder for. The decoder detects $w$ power units (P.U.) for active user or $\lambda$ P.U. for interferers, where the weight $w$ represents the number of occupied frequency bins in the user's encoder, and the in-phase cross-correlation $\lambda$ is the maximum number of common frequency bins occupied by any two codes of the family [10]. The remainder of the signal from the decoder is then transmitted to the subtractive decoder (s-Decoder) to cancel out signals with mismatched signatures, i.e., interferers. The s-Decoder contains only frequency bins from different interferers. After optical subtraction, the output from the s-Decoder is either zero P.U. for active user or $\lambda$ P.U. for interferers. This technique can be performed using low cost uniform FBGs to decode the received signal. This implies that, the interference signals are cancelled in the optical domain before the conversion of the signals to the electrical domain. As a consequence, the new SPD scheme alleviates both PIIN and MAI in the optical domain.

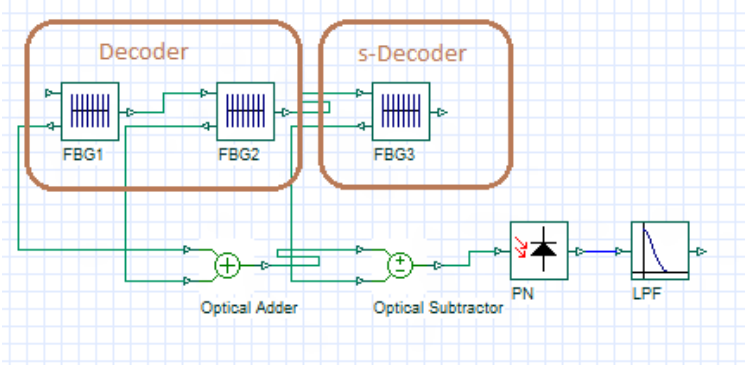

Figure 2 SPD detection

\section{NETWORK SiMULATION SETUP}

The simple schematic block diagram of three users SAC-OCDMA using EDW code with modifiedAND subtraction detection and SPD detection techniques are presented in Fig.3. As indicated in Fig.3, the transmitter of the SAC-OCDMA system using EDW code consists of one LED for each user at $622 \mathrm{Mbps}$ which is sliced to three wavelengths using wavelength division multiplexing (WDM) to generate the OCDMA codes.

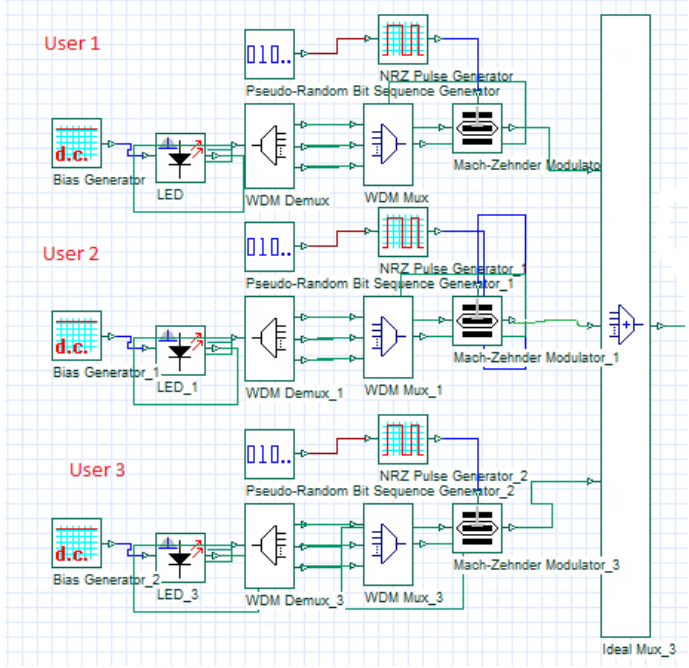


(a)

\section{(Q)}

Optical Fiber

(b)

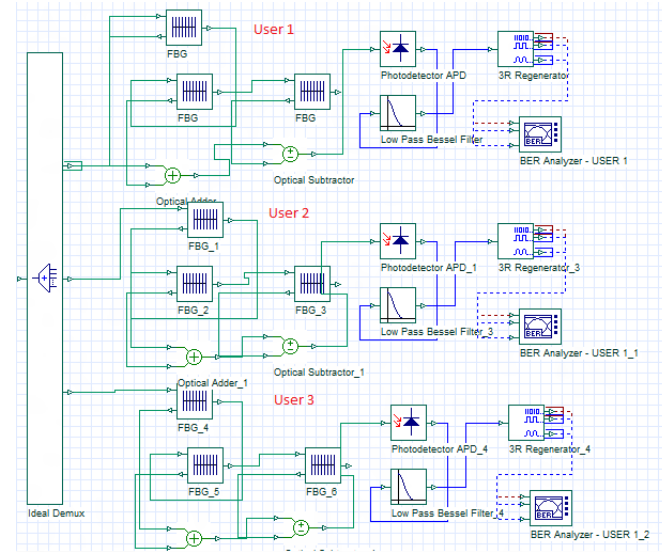

(c)

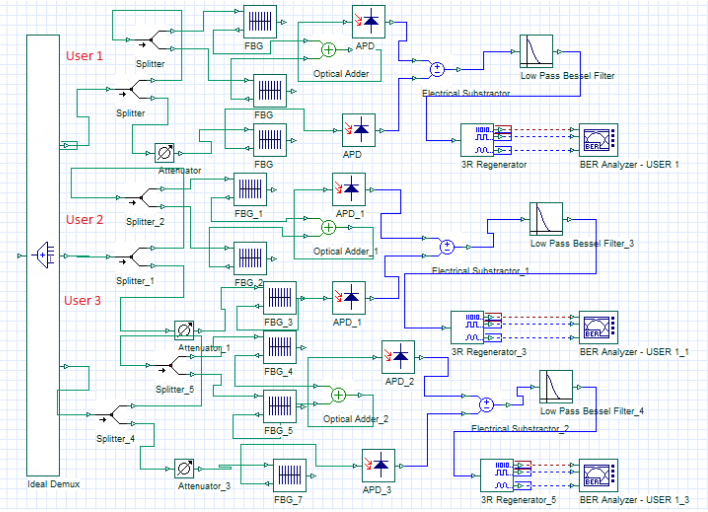

(d)

Figure 3 Block diagram of three users SAC-OCDMA (a) transmitter using EDW code (b) channel (c) receiver using SPD detection (d) receiver using modified-AND subtraction detection.

EDW codes are characterized by unity cross-correlation. EDW codes are denoted by $(N, w, \lambda)$, where $N$ is the code length which can be related to the number of users $K$ through [11],

$L=2 k+\frac{4}{3}\left[\sin \left(\frac{k \pi}{3}\right)\right]^{2} \frac{8}{3}\left[\sin \left(\frac{(k+1) \pi}{3}\right)\right]^{2}+\frac{4}{3}\left[\sin \left(\frac{(k+2) \pi}{3}\right)\right]^{2}$

Table 1 shows the EDW code sequences for $w=3$, that is used for the three users. The corresponding selected wavelengths are also shown in Table 1 .

Table 1 EDW code with $w=3$ and $N=6$ and the corresponding wavelengths.

\begin{tabular}{ccccccc}
\hline \hline $\mathbf{N}$ & $\mathbf{1}$ & $\mathbf{2}$ & $\mathbf{3}$ & $\mathbf{4}$ & $\mathbf{5}$ & $\mathbf{6}$ \\
\hline \hline Wavelengths (nm) & 1550 & 1550.8 & 1551.6 & 1552.4 & 1553.2 & 1554 \\
& 0 & 0 & 1 & 1 & 0 & 1 \\
Code sequences & 0 & 1 & 0 & 0 & 1 & 1 \\
& 1 & 1 & 0 & 1 & 0 & 0 \\
\hline \hline
\end{tabular}

The information signals are generated from the pseudo random bit generator with the non-return-tozero (NRZ) line coding before being modulated with the codes using an external Mach Zehnder modulator. Each chip has a spectral width of $0.8 \mathrm{~nm}$. The attenuation and the dispersion coefficients of the single mode fiber (SMF) at a wavelength of $1550 \mathrm{~nm}$ are $0.2 \mathrm{~dB} / \mathrm{km}$ and $17 \mathrm{ps} / \mathrm{km} / \mathrm{nm}$, respectively, according to the ITU-T G.652 standard. The signals are then combined using ideal multiplexer before launched to the SMF. 
The SAC-OCDMA receiver uses two different detection techniques which are the modified-AND subtraction detection and the SPD detection. In both detection techniques, FBGs use the same bandwidth but with different Bragg wavelengths as to decode the signal. The APD then converts the signal to the electrical domain. The resultant signal is filtered by fourth order Bessel low pass filter, which is used to reject noise and interference components that lie outside the information signal spectrum.

\section{RESULTS AND DISCUSSION}

The network simulation set up shown in Fig. 3 has been simulated using optisystem (version 7.0) with the parameters shown in Table 2. The optimum received power is evaluated for SAC-OCDMA system using EDW code with three users at $622 \mathrm{Mbps}$ and at different transmission distances and different APD gain for different detection techniques.

Figure 4 displays the relation between the fiber length and the measured BER for both the modifiedAND subtraction detection and SPD detection techniques.

Table 2 Typical parameters used for simulation.

\begin{tabular}{lc}
\hline \hline LED bandwidth & $30 \mathrm{~nm}$ \\
LED input power & $9 \mathrm{dBm}$ \\
Signal Data & $128 \mathrm{PN}$ sequence \\
Chip spectral width & $0.8 \mathrm{~nm}$ \\
Signal format & $\mathrm{NRZ}$ \\
External modulator extinction & $30 \mathrm{~dB}$ \\
Data rate & $622 \mathrm{Mbps}$ \\
FBGs reflectivity & 0.99 \\
Fiber dispersion, D & $17 \mathrm{ps} / \mathrm{nm}^{\mathrm{kmm}}$ \\
Fiber dispersion slope, S & $0.075 \mathrm{ps} / \mathrm{nm}^{2} / \mathrm{km}$ \\
APD gain & 10 \\
Dark current & $5 \mathrm{nA}$ \\
Thermal noise coefficient & $1.8 \times 10^{-23}$ \\
Receiver filter bandwidth & $0.75 \times \mathrm{Bit}$ Rate \\
Number of users & 3 \\
\hline \hline
\end{tabular}

Figure 4 clearly shows that, the SAC-OCDMA system when using SPD detection can travel a distance $120 \mathrm{~km}$ with BER $4.08 \times 10^{-12}$, while using the modified-AND subtraction detection the distance becomes $60 \mathrm{~km}$ with BER $2.43 \times 10^{-12}$. Therefore, the SPD detection technique is preferred.

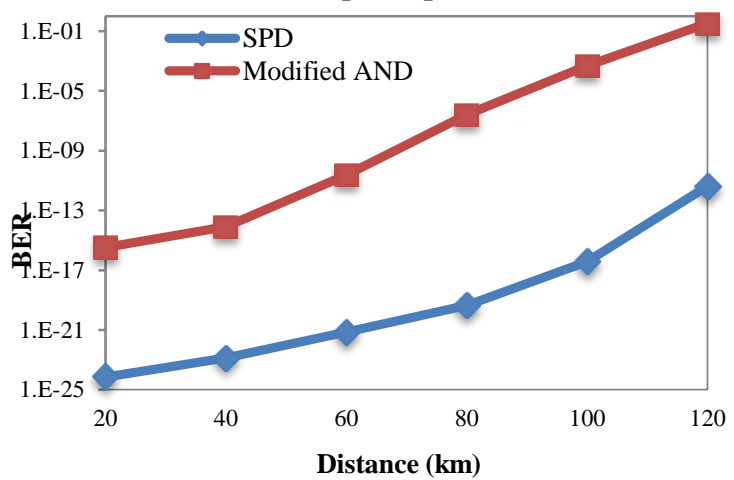

Figure 4 BER against fiber length using different detection techniques.

The received power is demonstrated against fiber length in Fig. 5 for both detection techniques. It is seen that, when the received power decreases with the fiber length in both techniques and the SPD detection technique yields the greater received power.

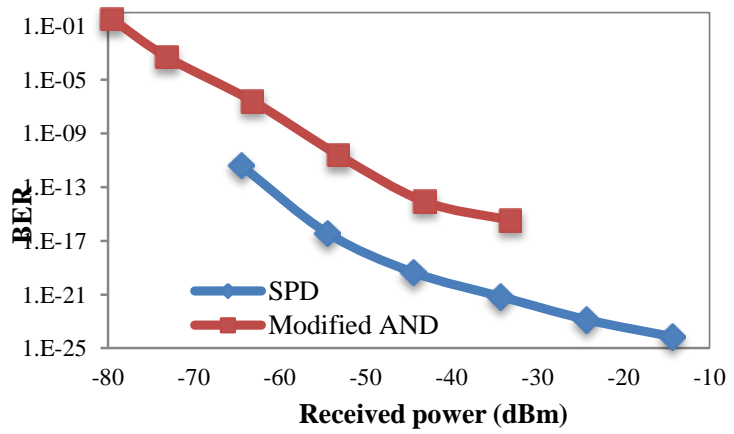

Figure 5 Received power against fiber length in different detection techniques. 
Figure 6 presents the relation between the received power and BER at different transmission distances for both detection techniques at constant bit rate $600 \mathrm{Mbps}$. One notices that, the optimum received power with standard error free transmission value (BER $\cong 10^{-9}$ ) is $-53 \mathrm{dBm}$ and $-64 \mathrm{dBm}$ for modified-AND subtraction and SPD detection, respectively. Referring to Fig. 5, this corresponds to distances $60 \mathrm{~km}$ and $120 \mathrm{~km}$, respectively.

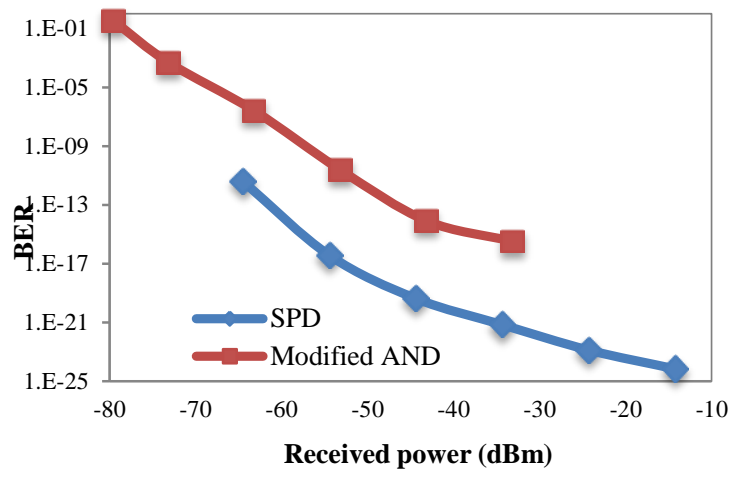

Figure 6 BER versus the received power in different detection techniques.

Figure 7 depicts the effect of APD gain on the received power for both detection techniques at 622 Mbps and at distance $20 \mathrm{~km}$. It is clear that, the received power increases with the APD gain. However, one cannot use higher gain APD because using higher gain produces bad BER [12].

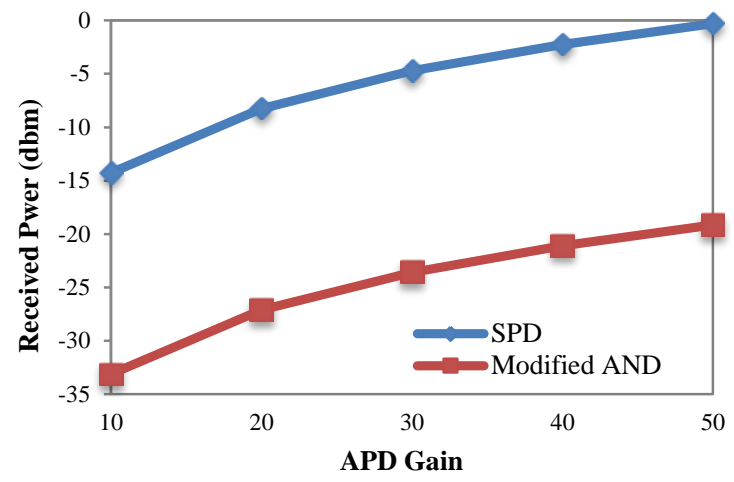

Fig. 7 Received power against APD gain in different detection techniques.

\section{CONCLUSION}

In-band transmission of OCDMA has been analyzed using numerical simulation to find out the optimum received power at $622 \mathrm{Mbps}$ and different transmission distances. Both modified-AND subtraction detection technique and SPD detection techniques with APDs are used at the receiver. EDW codes are used as signature addresses of the system. Different APD gains are used at a constant transmission distance. The results show that, modified-AND subtraction and the SPD detection techniques yield an optimum received power of $53 \mathrm{dBm}$ at $60 \mathrm{~km}$ and $-64 \mathrm{dBm}$ at $120 \mathrm{~km}$, respectively, at a transmitted power of $9 \mathrm{dBm}$. Considering these advantages, this implies the feasibility of the proposed system to be suitable for Fiber-to-the-Home (FTTH) access network to fulfill the consumer's demands.

\section{REFERENCES}

[1] N. Karafolas and D. Uttamchandani, Optical fiber code division multiple access network: A review, Optical Fiber Technology, 2(2), 149-168, 1996.

[2] H.W. Chen, G.C. Yang, C.Y. Chang, T.C. Lin and W. C. Kwong, Spectral efficiency study of two multirate schemes for asynchronous optical CDMA, Journal of Lightwave Technology, 27(14), 2771-2778, 2009.

[3] J.F. Huang, C.M. Tsai and Y.L. Lo, Compensating fiber gratings for source flatness to reduce multiple-access interferences in optical CDMA network coder/decoders, Journal of Lightwave Technology, 22(3), 739-745, 2004.

[4] F. A. Aziz and S. S. A. Obayya, Manchester-coded modified-legendre codes for spectral-amplitude coding-based optical codedivision multiplexing system, IET Optoelectron. , 5(2), 93-98, 2011.

[5] S. B. Ahmad Anas, M. K. Abdullah, M. Mokhtar and S. D. Walker, Multiple access interference elimination with enhanced chromatic dispersion tolerance in SAC OCDMA, IEICE Electron. Express., 5(16), 617-623, 2008.

[6] H. M. R. Al-Khafaji, S. A. Aljunid and H. A. Fadhil, Spectral efficiency analysis of bipolar spectral-amplitude coding optical codedivision multiple-access systems using different one-dimensional codes, IET Optoelectron., 6(5), 215-222, 2012.

[7] H. M. R. Al-Khafaji, S. A. Aljunid and H. A. Fadhil, Improved BER based on intensity noise alleviation using developed detection technique for incoherent SAC-OCDMA systems, Jornal of Modern Optics, 59(10), 878-886, 2012.

[8] I. S. Hmud, F. N. Hasoon, F. A. Hatim, A. Z. G. Zahid and S. Shaari, AND-subtraction detection modified SNR equation for optical CDMA systems in PON applications, Journal of Optica Applicata, 40(1), 49-55, 2010. 
[9] J.F. Huang and C.-C. Yang, "Reductions of multiple-access interference in fiber-grating-based optical CDMA network, IEEE Transactions on Communications, 50(10), 1680-1687, 2002.

[10] M. Rochette, S. Ayotte and L. A. Rusch, Analysis of the spectral efficiency of frequency-encoded OCDMA systems with incoherent sources, Journal of Lightwave Technology, 23(4), 1610-1619, 2005.

[11] Hasoon F. N., Mohamed Khazani Abdullah, Aljunid S. A. and Sahbudin Shaari, Construction of a new code for spectral amplitude coding in optical code-division multiple-access systems, Journal of Optical Engineering, 46(7), p. 75004, 2007.

[12] H. M. R. Al-Khafaji, S. A. Aljunid, Angela Amphawan, H. A. Fadhil and Anuar M. Safar, APD gain effect on SAC-OCDMA system using modified-AND detection technique, Journal of Theoretical and Applied Information Technology, 53(3), 467-471, 2013. 